\title{
Shape asymmetry of brain structures predicts dementia
}

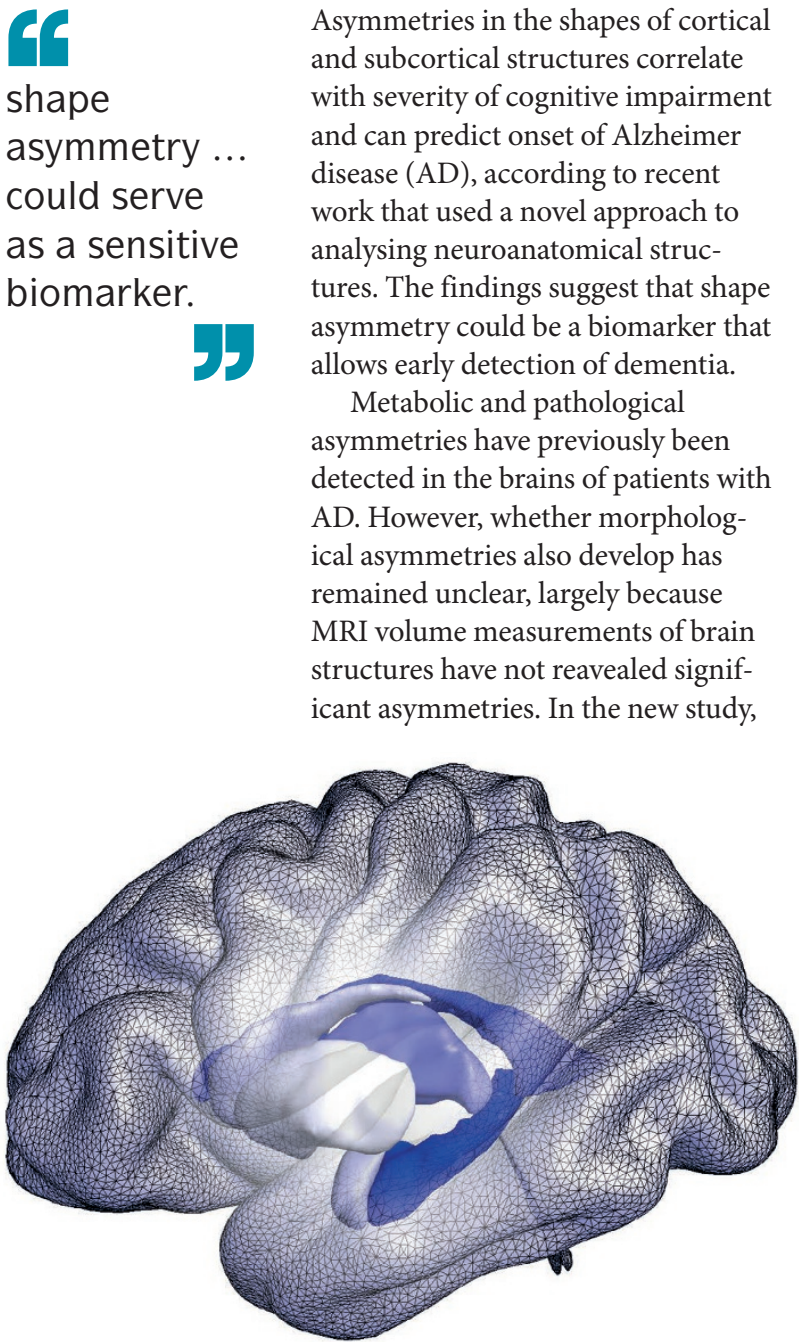

Asymmetries in the shapes of cortical and subcortical structures were significant in cognitive impairment and dementia. Image courtesy of C. Wachinger.
Christian Wachinger and colleagues have used a new, more sensitive imaging technique.

"We previously developed a new characterization of brain morphology based on shape descriptors, which we called the BrainPrint," explains Wachinger. "The BrainPrint captures the rich geometric information of brain structures, which is only crudely represented by commonly used volume measurements. We aim to use the advanced computational modelling in the BrainPrint to assist in the diagnosis of AD."

Wachinger and his team used BrainPrint to look at anatomical asymmetries in the brains of 697 people who had undergone at least three brain MRI scans as part of the Alzheimer's Disease Neuroimaging Initiative, which aims to determine whether serial MRI and PET can be used to measure disease progression. Participants were grouped according to cognitive status: cognitively normal, stable mild cognitive impairment (MCI), progression from MCI to $\mathrm{AD}$, and $\mathrm{AD}$.

Analysis showed that asymmetry of many brain structures increased with age, but greater effects in some structures accompanied cognitive impairment. In particular, asymmetries in the hippocampus and amygdala were significant in all groups with cognitive impairment, and a greater extent of asymmetry was seen with greater disease severity. Concurrent volume analysis failed to detect most of the asymmetries picked up by shape analysis.

The longitudinal design of the study also enabled the researchers to look for asymmetries that could predict progression from MCI to $\mathrm{AD}$. They found that shape asymmetry in the hippocampus, amygdala, caudate and cortex was predictive of progression. By contrast, volume asymmetry detected no predictors.

"The differentation between people with stable MCI and those who progress to $\mathrm{AD}$ is of high clinical relevance for selection into diseasemodifying therapies or drug trials," says Wachinger. "Our results indicate that neuroanatomical shape asymmetry of several subcortical structures could serve as a sensitive biomarker."

Wachinger says that the next steps are to further explore the relationship of shape asymmetries with established biomarkers of $\mathrm{AD}$, and learn more about the mechanisms that underlie the asymmetries.

ORIGINAL ARTICLE Wachinger, C. et al. Whole-brain analysis reveals increased neuroanatomical asymmetries in dementia for hippocampus and amygdala. Brain http://dx.doi. org/10.1093/brain/aww243 (2016) FURTHER READING Wachinger, C. et al. BrainPrint: a discriminative characterization of brain morphology. Neuroimage 109, 232-248 (2015) 